\title{
iReferrals: Time the referrals got intelligent
}

\author{
Author: Charlotte Masterton-Smith
}

\begin{abstract}
Aims
To improve the efficiency of the inpatient referral process to medical specialties.

Inpatient referrals to medical specialties at Kingston Hospital are done in many different ways with no unified system. Specialties use paper forms, magnets on whiteboards, phone referrals, Excel spreadsheets or even the fax machine. The highest volume of inpatient referrals to all medical specialties is from the acute assessment unit (AAU).

By creating a standardised electronic referral system to medical specialties, a referral can be made from, and reviewed by, the specialty team from any computer in the hospital. A triage system will be trialled to enable specialty teams to prioritise the order of referrals by urgent, routine or telephone advice only. We hope to improve efficiency when referring patients, become paper-free and reduce the number of bleeps and re-referrals made when the status of a referral is unknown. This system will also allow referrals to be more visible and improve the ability to audit them.
\end{abstract}

\section{Methods}

Junior doctors on AAU were surveyed about their current experiences of specialty referrals and suggestions were made as to how the system could be improved. An electronic referral form was created along with an outcome form for specialty teams to complete. All of these are synchronised with the electronic patient record system, so that when a patient is referred and reviewed, all of the information about that referral transfers automatically to the patient's notes.

Respiratory medicine and cardiology were identified as the two specialties with the largest volume of referrals and are participating in the pilot scheme for electronic referrals. Rigorous testing of this process was carried out with 'test' patients in the dummy electronic patient system. This enabled a real-time view of how referrals could be made and completed, identifying issues along the way.

The start of the pilot study has been publicised via the grand round meeting, email and with notices in the clinical areas.

\section{Results}

At present there are no results available as the electronic system for referrals to cardiology and respiratory medicine went live on

Author: Royal College of Physicians chief registrar, Kingston Hospital NHS Foundation Trust, UK
19 March 2018. We hope to have sufficient results from the first 1-2 months to fully present these soon.

\section{Conclusion}

If the use of electronic referrals proves to be successful it will be rolled out to other specialties in the hospital. Full conclusions will be available once data from the pilot scheme has been analysed.

\section{Conflict of interest statement}

None. 